[0212-7199 (2008) 25: 1; pp 23-26] ANALES DE MEDICINA INTERNA Copyright (C) 2008 ARAN EDICIONES, S.L.

AN. MED. InTERnA (Madrid) Vol. 25, N. ${ }^{\circ} 1$, pp. 23-26, 2008

\title{
Sarcoidosis sistémica necrotizante con afectación pulmonar y del sistema nervioso central
}

\author{
R. RÍOS FERNÁNDEZ, J. L. CALLEJAS-RUBIO, M. GUERRERO FERNÁNDEZ, \\ M. M. SERRANO FALCÓN, N. ORTEGO-CENTENO
}

Unidad de Enfermedades Sistémicas. Neurología. Anatomía Patológica. Hospital Clínico

San Cecilio. Granada

\begin{abstract}
NECROTIZING SYSTEMIC SARCOIDOSIS WITH PULMONARY AND CENTRAL NERVOUS SYSTEM INVOLVEMENT
\end{abstract}

\begin{abstract}
RESUMEN
La sarcoidosis es una enfermedad sistémica que se diagnostica con la presencia de granulomas no necrotizantes en una biopsia. Sin embargo, hay variantes como la sarcoidosis con granulomas necrotizantes o la sarcoidosis nodular en las que pueden aparecer zonas de necrosis que hacen que el diagnóstico diferencial con procesos infecciosos sea especialmente difícil.

Describimos un caso de un paciente, con afectación pulmonar y del $\mathrm{SNC}$, en quien la presencia de granulomas necrotizantes hizo orientar el diagnóstico inicialmente hacia una tuberculosis con el consiguiente retardo en la instauración del tratamiento específico.
\end{abstract}

PALABRAS CLAVE: Granuloma. Sarcoidosis. Sarcoidosis pulmonar. Tuberculosis. Vasculitis del sistema nervioso central. Vértigo.

\begin{abstract}
Sarcoidosis is a multisystemic disease which diagnosis depends on the presence of nonnecrotizing granulomas in the biopsy. However there are variants such as necrotizing sarcoidal granulomas or nodular sarcoidosis which have atypical findings and make difficult the differential diagnosis with other infectious processes.

We describe a case of a man who develops granulomas with extensive necrosis in a systemic sarcoidosis that affected the lung and the central nervous system. This finding made us to make the diagnosis of tuberculosis and delay the specific treatment
\end{abstract}

KEY WORDS: Sarcoidosis. Pulmonary sarcoidosis. Granuloma. Central nervous system vasculitis. Vertigo. Tuberculosis.

Ríos Fernández R, Callejas-Rubio JL, Guerrero Fernández, M, Serrano Falcón MM, Ortego-Centeno N. Sarcoidosis sistémica necrotizante con afectación pulmonar y del sistema nervioso central. An Med Interna (Madrid) 2008; 25: 23-26.

\section{INTRODUCCIÓN}

La sarcoidosis es una enfermedad multisistémica caracterizada por un proceso granulomatoso inflamatorio que afecta el pulmón (1) y otros órganos (2). Normalmente el diagnóstico microscópico de sarcoidosis se hace cuando el anatomopatólogo reconoce granulomas epitelioides no necrotizantes en la biopsia. No obstante, en algunos casos puede haber necrosis (3) y la sarcoidosis puede ser confundida con un proceso infeccioso. Describimos un caso de un paciente varón que desarrolló sarcoidosis con afectación pulmonar y del sistema nervioso central cuyo estudio histológico demostró granulomas con necrosis lo que contribuyó a dificultar el diagnóstico.

\section{CASO APORTADO}

El paciente era un varón de 31 años, trabajador del campo, fumador, con antecedentes de apendicectomía y absceso perianal que fue tratado quirúrgicamente. En el momento de la presentación, en enero de 2003, comenzó con mareos y pérdida de memoria. Los mareos consistían en vértigo rotacional episódicos con sensación de inestabi- lidad en la marcha, acompañados de sudoración, nauseas y vómitos. Tres meses más tarde tuvo una caída al suelo, con pérdida de conciencia, y movimientos tónico-clónicos, salivación excesiva, sin mordedura de lengua, con incontinencia urinaria y fecal, y con una obnubilación postcrisis de 48 horas. Se realizó en ese momento un electroencefalograma y un TAC craneal que fueron normales; fue diagnosticado de epilepsia y tratado con carbamazepina. Desde entonces, comenzó con debilidad en la pierna derecha, alteración en la marcha, y un importante empeoramiento del equilibrio, con frecuente caídas. También presentaba un habla no fluente, interrumpida y con déficits, confusión, pérdida de memoria episódica, dificultad para la comprensión de una conversación, empeoramiento de la escritura, problemas higiénicos e incontinencia de esfínteres. Su hermano, con quien él convivía, había sido diagnosticado recientemente de sarcoidosis con afectación pulmonar y de la piel. La exploración física era normal, sin fiebre ni signos de afectación pulmonar. La exploración neurológica mostraba un paciente alerta, orientado en espacio y tiempo, aunque bradipsíquico. La exploración de los pares craneales era normal, salvo nistagmo horizontal hacia el lado derecho. Las pupilas eran simétricas y reactivas. El fondo de ojo era normal. No había rigidez de nuca. Tenía una paresia grado 4+/5 en el brazo izquierdo y en ambas piernas. El tono era normal. Los reflejos estaban exagerados en ambas piernas. El reflejo cutáneoplantar era

Trabajo aceptado: 27 de agosto de 2007

Correspondencia: Raquel Ríos Fernández. Unidades de Enfermedades Autoinmunes. Hospital Clínico San Cecilio. Avda. Doctor Olóriz, 16.18012 Granada. e-mail: rriosfer@hotmail.com 
extensor en el pie izquierdo e indiferente en el derecho. La sensibilidad superficial y vibratoria era normal. El Romberg, inestable. A la marcha presentaba un aumento de la base de sustentación. Había un temblor de reposo y de intención en el brazo izquierdo y la realización de movimientos alternantes con ambas piernas estaba disminuida. Los resultados de laboratorio mostraron: GPT 48 U/1, GOT 30 U/l, fosfatasa alcalina $151 \mathrm{U} / \mathrm{l}$, GGT $36 \mathrm{U} / \mathrm{l}$; vit B12 292 pg/ml, ácido fólico $1 \mathrm{ng} / \mathrm{ml}$, proteinas totales $6.88 \mathrm{~g} / \mathrm{dl}$ (albúmina 52,7\%, alfa 1 $16,9 \%$, alfa $215 \%$, beta $9,2 \%$, gamma $16,1 \%$ ); IgG $1220 \mathrm{mg} / \mathrm{dl}$, ECA 60,8 U/l; serología para sífilis, salmonela, brucela, borrelia, citomegalovirus, Epstein-Barr virus, herpes simple, varicela, VIH y parotiditis eran negativos; anticuerpos anticardiolipinas y crioglobulinas negativos. El recuento linfocitario mostraba: CD3 $580 \mathrm{cel} / \mathrm{ul}$; CD4 $276 \mathrm{cel} / \mathrm{ul}$; CD8 $286 \mathrm{cel} / \mathrm{ul}$; B $308 \mathrm{cel} / \mathrm{ul}$; NK $168 \mathrm{cel} / \mathrm{ul}$. Se realizó una punción lumbar que mostró un líquido claro, con un recuento celular de 5 linfocitos $/ \mathrm{mm}^{3}$, glucosa $15 \mathrm{mg} / \mathrm{dl}$, proteinas totales 2,7 g/l, albumina 2,25 g/l, immunoglobulina $\mathrm{G} 38 \mathrm{mg} / \mathrm{dl}$, ECA 6,3 U/1, ADA 10 U/L. No había bandas oligoclonales ni síntesis de IgG intratecal. Los cultivos bacterianos y para hongos del líquido cefalorraquídeo y el test de tinta china fueron negativos. La citología del líquido cefalorraquídeo mostraba moderado número de leucocitos (polimorfonucleares y linfocitos). Se realizó un electroencefalograma que mostraba una actividad delta, más frecuente en el lado derecho, y unos potenciales evocados que presentaban anomalías en la respuesta auditiva y somatosensorial, con una alta amplitud en los potenciales somatosensoriales de los nervios medianos. Una resonancia magnética cerebral mostró lesiones focales bilaterales de 1-2 $\mathrm{mm}$ de diámetro, localizados en la sustancia blanca con señal hiperintensa en las secuencias FLAIR y T2. La mayoría de ellos eran periventriculares y estaban en la parte derecha de la protuberancia. Después de la administración de material de contraste se observó una captación meníngea que se extendía hacia la porción cervical (Fig. 1). Se realizó un TAC de senos que mostró una edematización de la mucosa de los senos etmoidales y un nivel hidro-aéreo en el seno maxilar derecho. La biopsia de la mucosa revelaba edema e infiltrado inflamatorio eosinofílico y no se observó granulomas. Cuando estudiamos el pulmón encontramos en la radiografía de tórax un infiltrado en el segmento S6 derecho con engrosamiento pleural. El TAC de tórax demostró múltiples nódulos mediastínicos e infiltrados broncoalveolares en ambas bases. Una gammagrafía con galio mostró áreas de hiperactividad en el hilio izquierdo pulmonar. El lavado broncoalveolar y las muestras de esputo fueron negativas para los bacilos ácido alcohol resistentes. Otras investigaciones para la tuberculosis incluyeron cultivos y PCR para micobacterias en esputo, que fueron negativas. El lavado broncoalveolar presentaba macrófagos y linfocitos B. Se realizó una biopsia del hígado que mostró infiltrados portales con actividad necroinflamatoria e histiocitos lobulillares. Después de una biopsia de pulmón abierta que mostró infiltrados granulomatosos necrotizantes (Fig. 2), el paciente comenzó inmediatamente tratamiento con fármacos tuberculostáticos (rifampicina, pirazinamida e isoniacida durante 2 meses, seguido de isoniacida y rifampicina durante ocho meses más) y esteroides (prednisona 60 $\mathrm{mg}$ /día) con intención de realizar un descenso progresivo. El paciente mejoró en la memoria, no tuvo más crisis epilépticas y desapareció la incontinencia urinaria. Sin embargo continuó con dificultad para la marcha con caídas frecuentes.

Después de terminar el tratamiento tuberculostático, y dos meses más tarde de suspender el tratamiento con esteroides, empeoró clínicamente apareciendo un síndrome de piernas inquietas, confusión, agitación nocturna, pérdida de memoria, inestabilidad en la marcha e incontinencia urinaria. Una nueva gammagrafía con galio-67 mostró captación en mediastino, ambos hilios pulmonares y parótida izquierda. El TAC de tórax mostró persistencia de los infiltrados en ambos campos pulmonares. Los ganglios hiliares y mediastínicos habían aumentado de tamaño. Se realizó un estudio de función pulmonar que mostró: VC 71\%; VEMS 61,7\%, VEMS/CV: $69,70 \%$, MMEF $75 / 25$ de $32,9 \%$, DLCO: $68,5 \%$, y $\mathrm{KCO}$ de $100,3 \%$.

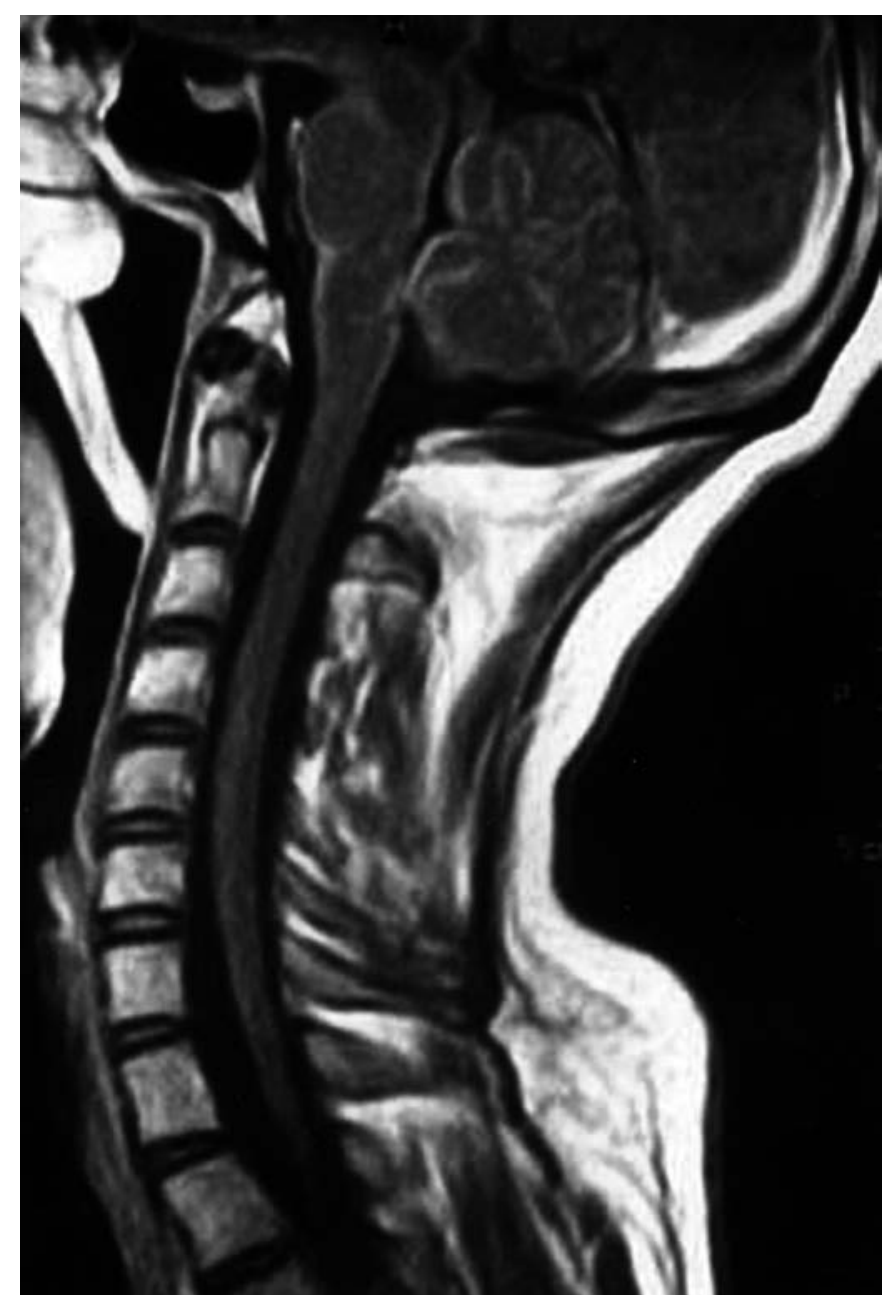

Fig. 1. Resonancia magnética cervical y cerebral donde se observa una captación meníngea tras la administración de material de contraste.

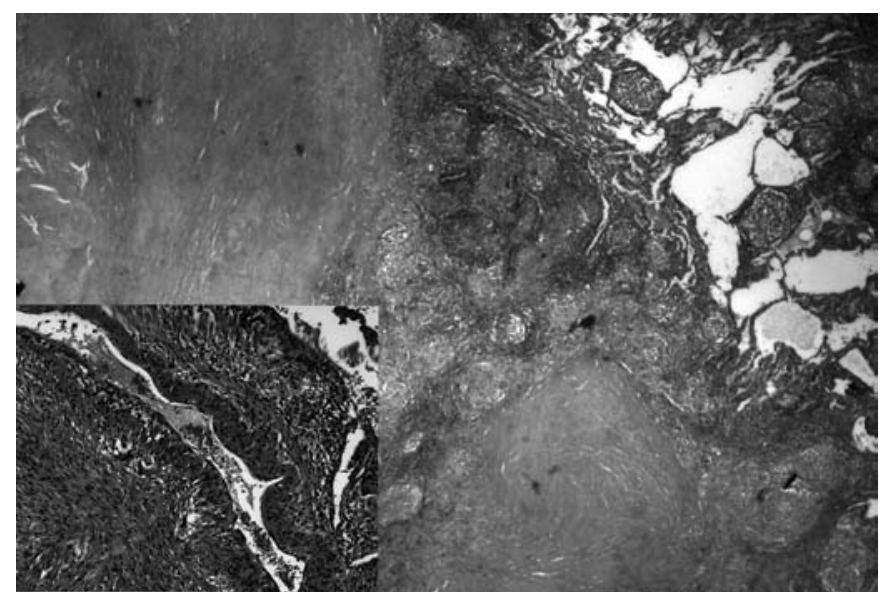

Fig. 2. Granulomas epiteliodes necrotizantes que afectan el tejido pulmonar. En el ángulo izquierdo se pueden observar pequeños granulomas epiteloides no necrotizantes. Los granulomas están presentes en las paredes vasculares (H\&E x 100).

Ante la posibilidad de una sarcoidosis necrotizante el paciente comenzó tratamiento con prednisona a dosis de $1 \mathrm{mg} / \mathrm{kg}$ de peso y metotrexato (15 mg/semana). La dosis de prednisona se redujo según respuesta clínica. Dos años y medio más tarde, el paciente continua 
con una buena evolución de las funciones cognitivas y de las actividades de la vida diaria; está recuperando su personalidad; mantiene un buen control de sus esfínteres y solamente persiste una mínima inestabilidad y debilidad en ambas piernas con caídas ocasionales. No presenta síntomas respiratorios y su función pulmonar es normal.

\section{DISCUSIÓN}

El diagnóstico de sarcoidosis es clinico-patológico. En un paciente con manifestaciones clínicas de sarcoidosis la identificación de granulomas epiteliodes no necrotizantes en más de un órgano es la clave para el diagnóstico. Pequeños focos de necrosis central o necrosis más extensa es poco frecuente y su hallazgo sugiere un proceso infeccioso o una sarcoidosis poco común como la sarcoidosis necrotizante. La sarcoidosis necrotizante (SN) fue descrita por primera vez por Liebow en 1973 (4). Si es una entidad o representa una variante de sarcoidosis es todavía material de debate. Sin embargo, es un hecho real que esta entidad se manifiesta con cavitaciones, una histología con vasculitis y marcada necrosis $(3,5)$. En nuestro caso, la necrosis detectada en la biopsia pulmonar nos sugirió el diagnostico de sarcoidosis necrotizante. En esta entidad, la necrosis es una consecuencia de la isquemia debido a la vasculitis (6). La patogénesis y la etiología de la variante necrotizante de la sarcoidosis, todavía no es bien conocida, aunque hay varios casos descritos en la literatura $(5,7,8)$. La aparición de la sarcoidosis en más de un miembro de una misma familia es un hecho que ya ha sido relatado por algunos autores $(9,10)$ y sugiere que hay una determinante común (ya sea genético o ambiental) que puede estar implicado. En la sarcoidosis es típica la afectación pulmonar, pero puede afectarse cualquier órgano (11). Entre las localizaciones poco frecuentes se encuentra el cerebro (12-14), el área retroorbital (15), los nervios craneales(8), la duramadre $(16,17)$, el riñón (18), el corazón (19), los ojos, la piel $(20,21)$, el hígado (22), el tracto gastrointestinal (23), las glándulas lacrimales (17) o los senos (7). La sarcoidosis primaria del sistema nervioso central es rara y se estima que puede ocurrir en aproximadamente el 5\% de todos los pacientes afectados con sarcoidosis $(15,24)$. Hay una predilección por la base del cerebro, pero también se pueden afectar otras partes del sistema nervioso central o periférico. Los hallazgos clínicos más frecuentes influyen meningitis, neuropatía craneal, disfunción pituitaria /hipotalámica, y déficit neurologicos secundarios al efecto masa intracraneal en el parénquima cerebral. (25). La resonancia magnética ha demostrado tener sensibilidad pero no especificidad en identificar la neurosarcoidosis $(8,26)$. La sarcoidosis meníngea se caracteriza por nódulos o placas focales o multifocales que captan contraste. Sin embargo, la tuberculosis, las infecciones bacterianas o fúngicas, la carcinomatosis, la leucemia o el linfoma producen la misma captación y son indistinguibles. La presencia de necrosis en las lesiones granulonatosas requiere la exclusión de infecciones. La tuberculosis produce un patrón histológico similar al de la sarcoidosis. Las infecciones fúngicas y por Pneumocystis Jiroveci también son causas de granulomas necrotizantes $(3,27)$. En nuestro caso, la larga duración de la enfermedad, la respuesta a esteroides, la negatividad de los cultivos y la falta de respuesta al tratamiento tuberculostático, hace menos probable que la etiología sea infecciosa. Solo el tratamiento con esteroides produjo una mejoría clínica y sintomática. El diagnóstico de la sarcoidosis, en muchos casos, es un diagnostico de exclusión. Los hallazgos de laboratorio como el ECA $(3,5,25)$ o el cociente CD4/CD8, pueden ayudar para el diagnóstico de sarcoidosis, pero ninguno de estos son específicos para su diagnostico (28), habiéndose abandonado el test de Kveim-Siltzbach.

El curso clínico de la SN normalmente es benigno, incluso con poco o ningún tratamiento $(5,29)$ pero, en la afectación del SNC (excepto la paresia del VII par craneal) no se produce una remisión espontánea y el tratamiento con esteroides e inmunospresores debe de comenzarse y continuarse durante años con la finalidad de prevenir una afectación permanente (30). El principal tratamiento para la neurosarcoidosis son los esteroides. La radioterapia y el tratamiento inmunosupresor, particularmente el metotrexato, la azatioprina, y la ciclosporina se han usado en casos resistentes $(25,31)$. En nuestro caso la asociación de metotrexato permitió un buen control de la enfermedad y el disminuir rápidamente la dosis de glucocoticosteroides.

En conclusión, la SN del SNC es una entidad de características histológicas específicas que pueden dificultar su diagnóstico. El conocimiento de esta entidad puede facilitar un diagnóstico precoz que permita instaurar un tratamiento adecuado a fin de que se instaure un déficit neurológico permanente.

\section{Bibliografía}

1. Cuadra Llopart L, Barreiro López B, Forcada Guiu P. Necrotizing sarcoid granulomatosis. Arch Bronconeumol 2005; 41: 355-6.

2. Newman LS, Rose CS, Maier LA. Sarcoidosis. N Engl J Med 1997; 336: 1224-34.

3. Gal AA, Koss MN. The pathology of sarcoidosis. Curr Opin Pulm Med 2002; 8: 445-51.

4. Liebow AA. The J. Burns Amberson lecture: Pulmonary angiitis and granulomatosis. Am Rev Respir Dis 1973; 108: 1-17.

5. Heinrich D, Gordjani N, Trusen A, Marx A, Hebestreit H. Necrotizing sarcoid granulomatosis: A rarity in childhood. Pediatr Pulmonol 2003; 35: 407-11.

6. Popper HH, Klemen H, Colby TV, Churg A. Necrotizing sarcoid granulomatosis is it different from nodular sarcoidosis? Pneumologie 2003; 57: 268-71.

7. Hasni SA, Gruber BL. Sarcoidosis presenting as necrotizing sinus des- truction mimicking Wegener's granulomatosis. J Rheumatol 2000; 27 : 512-4.

8. Jennings JW, Rojiani AM, Brem SB, Murtagh FR. Necrotizing neurosarcoidosis masquerading as a left optic nerve meningioma: Case report. AJNR Am J Neuroradiol 2002; 23: 660-2.

9. Rybicki BA, Iannuzzi MC, Frederick MM, Thompson BW, Rossman MD, Bresnitz EA, et al. Familial aggregation of sarcoidosis. A casecontrol etiologic study of sarcoidosis (ACCESS). Am J Respir Crit Care Med 2001; 164: 2085-91.

10. Pietinalho A, Ohmichi M, Hirasawa M, Hiraga Y, Lofroos AB, Selroos O. Familial sarcoidosis in Finland and Hokkaido, Japan-a comparative study. Respir Med 1999; 93: 408-12.

11. Churg A, Carrington CB, Gupta R. Necrotizing sarcoid granulomatosis Chest 1979; 76: 406-13.

12. Brevet F, Hachulla E, Courtin P, Gosselin B, Wurtz A, Hatron PY, et al 
Systemic form of a sarcoid-like necrotizing granulomatosis with ocular, pulmonary and cerebral involvement. Rev Med Interne 1993; 14: 243-8.

13. Stricklang-Marmol LB, Fessler RG, Rojiani AM. Necrotizing sarcoid granulomatosis mimicking an intracranial neoplasm: clinicopathologic features and review of the literature. Mod Pathol 2000; 13: 909-13.

14. Caplan L, Corbett J, Goodwin J, Thomas C, Shenker D, Schatz N. Neuro-opthmologic signs in the angiitic form of neuro-sarcoidosis. Neurol 1983; 33: 1130-5.

15. Dykhuizen RS, Smith CC, Kennedy MM, McLay KA, Cockburn JS, Kerr KM. Necrotizing sardoid granulomatosis with extrapulmonary involvement. Eur Respir J 1997; 10: 245-7.

16. Tobias S, Prayson RA, Lee JH. Necrotizing neurosarcoidosis of the cranial base resembling an en plaque sphenoid wing meningioma: Case report. Neurosurgery 2002; 51: 1290-4.

17. Singh N, Cole S, Krause PJ, Conway M, García L. Necrotizing sarcoid granulomatosis with extra-pulmonary involvement. Clinical, pathologic, ultrastructural, and immunologic features. Am Rev Respir Dis 1981; 124: 189-92.

18. Ito Y, Suzuki T, Mizuno M, Morita Y, Muto E, Ichida S, et al. A case of renal sarcoidosis showing central necrosis and abnormal expression of angiotensin converting enzyme in the granuloma Clin Nephrol 1994; 42: 331-236.

19. Gibbs AR, Williams WJ, Kelland D. Necrotizing sarcoid granulomatosis: A problem of identity. A study of seven cases. Sarcoidosis 1987; 4: 94-100.

20. Shirodaria CC, Nicholson AG, Hansell DM, Wells AU, Wilson R. Necrotizing sarcoid granulomatosis with skn involvement. Histopathology $2003 ; 43: 91-3$.
21. Kuramoto Y, Shindo Y, Tagami H. Subcutaneous sarcoidosis with extensive caseation necrosis. J Cutan Pathol 1988; 15: 188-90.

22. Devaney K, Goodman ZD, Epstein MS, Zimmerman HJ, Ishak KG. Hepatic sarcoidosis clinicopathologic features in 100 patients. Am J Surg Pathol 1993; 17: 1272-80.

23. Le Gall F, Loeuillet L, Delaval P, Thoreux PH, Desrues B, Ramee MP. Necrotizing sarcoid granulomatosis with and without extrapulmonary involvement. Pathol Res Pract 1996; 192: 306-13.

24. Delaney P. Neurologic manifestations in sarcoidosis: review of the literature, with a report of 23 cases. Ann Intern Med 1977; 87: 336-45.

25. Sharma OP. Neurosarcoidosis: a personal perspective based on the study of 37 patients. Chest 1997; 112: 220-8.

26. Sherman JL, Stern BJ. Sarcoidosis of the CNS: Comparison of unenhanced and enhanced MR images. AJNR Am J Neuroradiol 1990; 11: 915-23.

27. Ulbright TM, Katzenstein AL. A solitary necrotizing granulomas of the lung: Differentiating features and etiology. Am J Surg Pathol 1980; 4: 13-28.

28. Spiteri MA, Gledhill A, Campbell D, Clarke SW. Necrotizing sarcoid granulomatosis. Br J Dis Chest 1987; 81: 70-5.

29. Rodríguez-Gómez F, Borrero Martín JJ, Merino Muñoz D, Pujol de la Llave E. Granulomatosis sarcoidea necrosante autolimitada. Med Clin (Barc) 2003; 120: 676-9

30. Marangoni S, Argentiero V, Tavolato B. Neurosarcoidosis: clinical description of 7 cases with a proposal for a new diagnostic strategy. J Neurol 2006; 253: 488-95.

31. Beach RC, Corrin B, Scopes JW, Graham E. Necrotizing sarcoid granulomatosis with neurologic lesions in a child J Pediatr 1980; 97: 950-953. 